\title{
Assessment of Pesticides Use and its Economic Impact on the Apiculture Subsector in Selected Districts of Amhara Region, Ethiopia
}

\section{Desalegn Begna*}

Holeta Bee Research Center, Holeta, Ethiopia

\begin{abstract}
Apiculture is one of the most widely spread agricultural subsectors making substantial contribution to household food security through providing income, quality foods and assets to the rural beekeepers in Ethiopia. Ethiopia is endowed with immense apicultural resources and long standing traditional beekeeping practices.

Recently, there is a growing pesticides grievance on honeybee population and their products decline with considerable economic impacts on beekeepers. To ascertain the gripe, field assessment was conducted on pesticides use and its economic impacts on the apiculture subsector in three districts of Amhara region (Dangila, Guangua and Mecha) in April 2014. Random household survey was conducted on a total of 270 respondents (90 per district) of which 137 were beekeepers and 133 none beekeepers.

From the total respondents, 147 uses pesticides and Dimethoate $40 \%$ EC, Ethiolathion $50 \%$, Karate 5EC, 2,4-D were the most often used pesticides. March, June, July and September are identified as pesticides applications months. From the total pesticides users (147) $114(78 \%)$ apply before flowering stage, 25 both before and during flowering stages and the rest applies whenever they feel pests and/ or weeds occur. Besides, more than $60 \%$ of the respondents know pesticides kill honeybees and results in dwindling and absconding. Three years trends analysis of honeybee colony number and honey yield indicated dramatic decline mainly attributed to indiscriminate applications of pesticides. This assessment revealed indiscriminate uses of pesticides caused fatalities on 22987 honeybee colonies and incurred economic loss amounting USD 819291.37.

This study is appropriate and timely to develop and implement effective development and extension strategies to minimize and/or control the ill effects of accidentally using pesticides.
\end{abstract}

Keywords: Apiculture; Beekeeping; Dimethoate 40\% EC; Economic impacts; Extension

\section{Introduction}

With about seven million honeybee populations, the Ethiopia's annual honey and beeswax production is estimated to be over 54,000 and 5000 tons, respectively (MoA 2013). With this, the country is ranking ninth highest honey producer in the world and the leading producer of honey and beeswax in Africa [1,2].

Beekeeping is significantly contributing to the beekeeper's livelihood and to the country's economy. To this fact, about 1.5 to 1.8 million households earn various levels of income a year from beekeeping. Tej (mead) to which the major proportion of local honey goes is with high calorie supplements to traditional diets providing significant additional rural employment and incomes. Although not quantified for local conditions, beekeeping through pollination is highly contributing to crop yield, quality of environment and biodiversity conservation. The experience in the United States indicates that the value of pollination services provided by honeybees is estimated at 14.6 billion dollars annually [3-5].

Recently the global honeybees have presented a decline with considerable economic impacts and beekeepers. Abiotic stress from the lethal effects of pesticides is currently being scrutinized as a contributing factor to poorly understood bee colony losses [6]. Pesticides are a class of chemicals or biological agent with properties designed to deter, kill, incapacitate, or otherwise limit damage by pests [6].

The introduction of pesticide in Ethiopia to control agricultural pests' dates back to the 1960's [7]. Although, the volume fluctuates across the pesticide types, the country on the average imports 3346.32 metric tons of pesticides annually [8]. Using pesticides is widely spread following modern agriculture and areas with high crop framing parts of Ethiopia are yearly receiving different types and amounts of pesticides.

Recently, there is growing consensus that pesticides have killed honeybees and their food source plants and resulted in bee death and their products declines $[9,10]$. However, the available information on the side effects of pesticides under local situations are little and incomplete $[9,10]$ as well as remaining obscure. Therefore, the main purpose of this assessment was to cut through the confusion and provide an up-to date and balanced explanation on the side effects of pesticides on honeybees and their products.

\section{Materials and Methods}

The study was conducted in three districts, namely Guangua, Dangila and Mecha of Amhara regional state. Three Kebele Administrations (KAs) from each district, in total nine KAs were selected as survey points. From each KA 30 households in total 90 per district and 270 from the three districts were interviewed on prestructured questionnaires for their attitudes and knowledge on the side effects of pesticides on bees' and their products. Also checklists were prepared and framers' perceptions were collected through focused group discussions from each KA.

In addition, pesticides and veterinary drug shops were included in the assessments. The selections of KAs were done based on the potential they have for beekeeping (forest cover/bee forage, existing practices etc), their access to road and service providers, and practices of irrigation for high value crops and pesticides effects. Purposive sampling was used

*Corresponding author: Desalegn Begna, Holeta Bee Research Center, P.O.Box 22, Holeta, Ethiopia, Tel: +251911353035; Fax: +39 0577 232930; E-mail desalegnbegna@yahoo.co.uk

Received October 24, 2014; Accepted January 18, 2015; Published January 25 2015

Citation: Begna D (2015) Assessment of Pesticides Use and its Economic Impact on the Apiculture Subsector in Selected Districts of Amhara Region, Ethiopia. J Environ Anal Toxicol 5: 267. doi:10.4172/2161-0525.1000267

Copyright: (c) 2015 Begna D. This is an open-access article distributed under the terms of the Creative Commons Attribution License, which permits unrestricted use, distribution, and reproduction in any medium, provided the original author and source are credited. 
in identifying the districts and KAs while random sampling was used in selecting sample enumeration areas and respondents from each household. All the collected data were properly coded and entered into an Excel spreadsheet, cleaned, verified and analyzed. Tables and graph were used with respect to the given variables, and the analyzed data and the major findings were reported as district's information.

\section{Results}

In this assessment different brands of pesticides were listed by the respondents and information was cross-checked through the survey made to pesticides. The most used brands of pesticides were Malathion $50 \%$, phenetratite $50 \%$ Ethiothoate $40 \%$, Agrothoate $40 \%$, Diazion $60 \%$ EC, Dimethoate $40 \%$ EC, Ethiolathion 50\% EC or Malathion, Karate 5EC, and herbicides like 2,4-D Amine, Zura, Diazion60\% EC, AgroThoate40\%, Etho-Thoate40\%, Hepta clore, Phenetratite50\%, Daconil, Diasnol, Primagram, Roundup, Agroset, Glycell and Terminator, which are concurrent to the brand type listed by Gizachew [8]. About $61 \%$ of the pesticides used by the farmers were identified as herbicides, $21 \%$ insecticides and $18 \%$ both types (Table 1 ).

Also, from the total of 270 beekeepers interviewed, 147 (54\%) are identified as pesticides users. However, the number of farmers using pesticides varied among the districts and the number was high in Guangua (Table 2).

Also following increased demand for pesticides use in the agriculture and flower farms sectors, the number of pesticides importers and distributors has increased in Ethiopia [8]. It is recognized that $53.4 \%$ of pesticides suppliers are private in small shops, $25.3 \%$ farmer cooperative, $5.5 \%$ companies (AISCO, GUNA, and EAL).

This assessment showed more than $80 \%$ of pesticides applications are done in March, June, July, September and October and $64.4 \%$ of the users' prefer 6:00-9:00am as appropriate spray time. However, through focused group discussions it was disclosed as applications times are fixed by Knapsack renters. It is only few farmers who own Knapsack and hence, these owners make rental schedule and also perform the spay works by themselves at their convenient times. Also, about $58 \%$ of the respondents apply only once, $36 \%$ twice and only $6 \%$ apply up to three times. The number of respondents applying two and three times is high in Mecha district.

According to this study, from the total 147 pesticides users, 114 apply pesticides before the crops bloom, 25 both before and during the crops in bloom and only three farmers from Mecha district practices presowing and pre-crops emergencies pesticides applications (Table 3).

From the total 147 farmers recorded for using pesticides, 125 apply in liquid (emulsified), 13 in powder and eight both in liquid and powder forms. The number of farmers using pesticides as powder is higher in Guangua district (10 from the total 13 in the district). Also, it has been identified that about $97 \%$ the farmers in the study areas uses manual (knapsack) mode of spray.

According to this study, more than $74 \%$ of the beekeepers didn't locate their apiary by considering the distance between apiary site and farm land that is yearly receiving pesticides. During focused group discussions, it was stated as some none-beekeeper crop growers do pesticide spay jealously.

Losses incurred due to pesticides' side effects on honeybees was estimated by analyzing the number of honeybee colonies dead, absconded and dwindled in 2013 (Figure 1). In total, 5209, 12109 and 5669 bee colonies were recorded died, absconded and dwindled in the three districts, respectively. However, there are variations between the number of honeybee colonies died, absconded and dwindled, and variations are apparent between absconded honeybee colonies and the rests. Likewise, there are high variations among the districts for the same parameters. In particular, the number of died, absconded and dwindled bee colonies were high in Guangua district (Figure 1).

Subsequent analysis of financial loss incurred due to the dead, absconded and dwindled honeybee colonies was estimated to a total of about 819291.4 USD (Table 4). Aligned with high number of absconded bee colonies, the estimated financial loss through this trajectory alone is quite substantial. Similarly, there are differences among the districts in financial fatalities and Guangua district is with the highest one.

It is known that about $69 \%$ of the respondents have got an extension services and are already aware of when and how to properly use pesticides without producing effects on the environment and honeybees. As well, visit to retailers shops demonstrated presences basic information on users' manual (information) on the labels of some pesticides.

\section{Discussion}

Honeybees are a vital part of global agricultural system through providing pollination services to many species of crops. Estimations are showing the annual value of honeybee pollination is more than 14.6 billion dollars in the US [5]. On the other side, increasing agricultural products to feed the ever increasing global population necessitated using of different kinds of pesticides to protect the crops from diseases and pests attacks. In the due course, honeybees and their products were subjected to pesticides ill effects. This loss of bees and their products decline due to the increased use of pesticides has caused widespread concern in many countries [11]. Therefore, this increased use of pesticides aligned with increased demand for bees' products and their pollination services have all added to the importance of protecting bees from pesticides.

In line with increased demand for food self sufficiency and high

\begin{tabular}{|c|c|c|c|c|c|}
\hline Types of pesticides & \multicolumn{3}{|c|}{ District and number of } & Total & Proportion (\%) \\
& respondents & & \\
\hline & Mecha & Dangla & Guangua & & \\
\hline Insecticide & 14 & 7 & 10 & 31 & 21 \\
\hline Herbicide & 22 & 19 & 48 & 89 & 61 \\
\hline $\begin{array}{c}\text { Both Insecticide and } \\
\text { Herbicide }\end{array}$ & 18 & 5 & 4 & 26 & 18 \\
\hline Total & 54 & 31 & 62 & 147 & 100 \\
\hline
\end{tabular}

Table 1: Pesticides categories and number of respondents using them

\begin{tabular}{|c|c|c|c|c|}
\hline \multirow{2}{*}{ Responses } & \multicolumn{2}{|c|}{ District and number of respondents } & \multirow{2}{*}{ Total } \\
\cline { 2 - 5 } & Mecha & Dangla & Guangua & \\
\hline Yes & 54 & 31 & 62 & 147 \\
\hline No & 36 & 59 & 28 & 123 \\
\hline
\end{tabular}

Table 2: Number of respondents using pesticides

\begin{tabular}{|l|c|c|c|c|}
\hline \multirow{2}{*}{ Crop stages } & \multicolumn{3}{c|}{$\begin{array}{c}\text { District and number of } \\
\text { respondents }\end{array}$} & Total \\
\hline & Mecha & Dangla & Guangua & \\
\hline Pre-sowing and pre-emergence & 3 & 1 & - & 4 \\
\hline Before flowering & 37 & 23 & 54 & 114 \\
\hline Before flowering and at fruiting & 12 & 6 & 7 & 25 \\
\hline Whenever pest occur for insects & 2 & 1 & 1 & 3 \\
\hline Total & 54 & 31 & 62 & 147 \\
\hline
\end{tabular}

Table 3: Stage of crop when pesticides are used 
Page 3 of 4

\begin{tabular}{|c|c|c|c|c|c|c|c|c|c|c|}
\hline \multirow[b]{2}{*}{ District } & \multirow[b]{2}{*}{$\begin{array}{l}\text { Mean yield/ } \\
\text { Year (KG) }\end{array}$} & \multirow[b]{2}{*}{$\begin{array}{c}\text { Price of } \\
\text { honey/Kg } \\
\text { (USD) }\end{array}$} & \multirow[b]{2}{*}{$\begin{array}{c}\text { Mean price } \\
\text { of a bee } \\
\text { colony (USD) }\end{array}$} & \multicolumn{3}{|c|}{ Number of bee colonies } & \multicolumn{3}{|c|}{ USD losses incurred as a results of } & \multirow{2}{*}{$\begin{array}{c}\text { Total (USD) } \\
(A+B+C)\end{array}$} \\
\hline & & & & Died & Left & Dwindled & $\begin{array}{l}\text { bee colonies } \\
\text { dead } \\
\text { (A) }\end{array}$ & $\begin{array}{l}\text { bee colonies } \\
\text { absconded } \\
\text { (B) }\end{array}$ & $\begin{array}{l}\text { bee colonies } \\
\text { dwindled } \\
\text { (C) }\end{array}$ & \\
\hline Mecha & 21 & 2.3175 & 35 & 44 & 66 & 529 & 3655 & 2363 & 1226 & 7244 \\
\hline Dangila & 19 & 2.5725 & 35 & 610 & 1322 & 305 & 50673 & 46334 & 785 & 97792 \\
\hline Guangua & 19 & 1.995 & 35 & 4555 & 10721 & 4835 & 329335 & 375275 & 9646 & 714255 \\
\hline Mean & 20 & 2 & 35 & 1736 & 4036 & 1890 & 127887 & 141324 & 3886 & 273097 \\
\hline
\end{tabular}

Table 4: Incurred economic losses adhering to pesticides applications (USD)

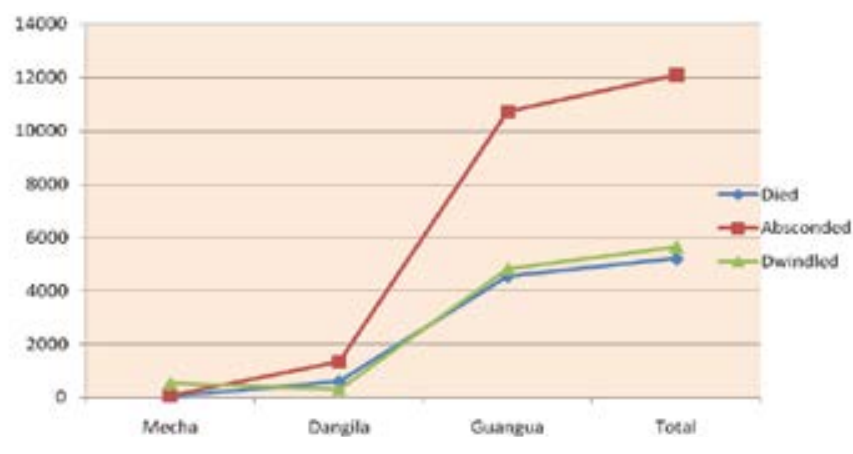

Figure 1: Pesticides sustained losses by case category across the districts

value crops, the farm lands are expanding at fast rate and so do the use of different pesticides. Following the demand of pesticides in the agriculture and flower sectors, the used quantities of pesticides have grown from 3327.7 metric tons in 2006 to 4211.5 in 2010 metric tons annually. On the average the country annually uses 3346.32 metric tons of pesticides [8].

In the assessment areas, different brands of pesticides are used at the same time and/or different seasons. This suggests the intermittent occurrences of different crop pests that necessitate the applications of pesticides with different brands. Also, the identification of herbicides as most often used pesticide by $98 \%$ of the applicators shows that crop weeds are the most common pest of the areas. Furthermore, this suggests that herbicides are becoming the best alternative when labor is in short of and expensive to remove the weeds by hand.

Although the majority of the farmers (64.4\%) have chosen early morning (before the start of bees' normal activities) as appropriate time to spray, the time is fixing Knapsack renters. This suggests requisite pesticide users' capacity building scheme so that they can have their own Knapsack either in group or individually to abide to their application schedules. The slight elevation of pesticides application frequencies in Mecha district might be attributed to high irrigation practices and presence of stimulant plant "Chat" known as Catha edulis plant that requires often pesticide spray to control the reoccurring pests. The high preferences of manual (knapsack) application is mainly attributed to fragmented and small holding size (0.71-1.5 ha) [2]. Furthermore, the failing of the beekeepers to locate apiaries in considering distances from the farm that is yearly receiving pesticides also emerges from current land tenure system that permit crop farming and beekeeping to take place adjoining. Moreover, the effects of pesticides due to none beekeepers indiscriminate uses and the jealously actions are showing absences of governing policy that put in place forcing measures so that the criminals can be penalized.

\section{Conclusion}

In the areas, most of the farmers extensively apply different brands of pesticides. Herbicides are mainly used at different times of the year to control weeds before sowing and in the crops. Most of the pesticides are accessed through informal suppliers that would not give advices on the proper handling and safe uses. Moreover, most of pesticides users have no knapsack and the knapsack renters determine the application time and techniques. As a result, pesticides have caused and been causing considerable effects in killing honeybees and their products decline. To this evident, on the average, 1736, 4036 and 1890 honeybee colonies are dying, absconding and dwindling every year from each district, respectively. Adhering to the effects on honeybees, the average economic loss incurred through their products is estimated to 273097 USD per year per district. For this reasons, beekeepers identified indiscriminate applications of pesticides are as major constraints of beekeeping developments in their areas.

In conclusion, the study availed evident and balanced information on the side effects of pesticides on honeybees and their products that is leading to developing strategies, policy and practices towards mitigating the risks.

\section{Recommendations}

Mitigating damage of pesticide use to honeybees is the responsibility of all parties involved and requires concerted effort to minimize the risk. Hence, based on this study the below are presented as possible recommendations, which are aimed at minimizing the ill effects of pesticides on honeybees and their products.

Manual should be developed and farmers must be educated on how to use label instructions and put into practice safety measures like not to spray on blooming crops, to keep bee colony away from the farm receiving pesticides, adjust the application time to late evening etc.

Regulatory body that oversees the total supply, transportation, storage, appropriateness etc of pesticides at all levels should be in place.

Crops weed management practices known by the community like hand weeding should be capitalized at least for two reasons: to protect bees and the environment; and to ensure the products are natural.

Initiating community based bylaws that give full right of supervise and corrective measures to the community.

The Amhara regional state should have its own context beekeeping development strategy and regional apiculture resources development and protection policy cascaded from the national one.

Comprehensive research into the effects of pesticides on honeybees and their products decline to which this study targeted to contribute is important.

\section{Acknowledgement}

This work is fully supported by OXFAM GB based in Ethiopia. Hence, I would like to express my sincere gratitude to OXFAM GB for covering all the study costs and also for allowing me to share this information publicly. As well, I am thankful to 
Citation: Begna D (2015) Assessment of Pesticides Use and its Economic Impact on the Apiculture Subsector in Selected Districts of Amhara Region, Ethiopia. J Environ Anal Toxicol 5: 267. doi:10.4172/2161-0525.1000267

Page 4 of 4

many people from the various governmental and nongovernmental organizations for supporting the work in one way or another

\section{References}

1. MoA (2013) Ministry of Agriculture and rural development annual report.

2. CIAFS (2012) The capacity to Improve Agriculture and Food security (CIAFS) The World Market For Honey Market Survey.

3. Christian HK, Gregory H, Rick EF (2012) Protecting honeybees from pesticides.

4. Roger AM, Nicholas WC (2000) The Value of Honeybees as Pollinators of U.S. crops in 2000. Cornell University Ithaca - New York. Bee Culture 128.

5. Malcolm TS (2011) Protecting Honeybees from Pesticides. Institute of Food and Agricultural Sciences, University of Florida, Gainesville, FL 32611.

6. Marion and Bethany (2011) Assessing the Risks of Honeybee Exposure to Pesticides. American Bee Journal and in Bee Culture.

7. EPA (2004) Federal Environmental Protection Authority, Environmenta Impact Assessment Guideline on Pesticides, Addis Ababa, Ethiopia.

8. Gizachew Assefa (2011) Pesticide use in Ethiopia. Ministry of Agriculture Addis Ababa.

9. Kerealem E, Tilahun G, Preston TR (2009) Constraints and prospects for Apiculture Research and Development in Amhara region, Ethiopia. Livestock Research for Rural Development.

10. Marta Zelalem Mengistu, Tariku JB (2014) Beekeeping in Ethiopia: A case of agrochemical use in West Gojjam Zone.

11. Erik Stokstad (2013) Pesticides under Fire for Risks to Pollinators. Science 340: 674-676. 